\title{
Switching from Sitagliptin to Alogliptin under Treatment with Pioglitazone Increases High Molecular Weight Adiponectin in Type 2 Diabetes: A Prospective Observational Study
}

\author{
Akinori Tokito1, Nobuyuki Koriyama², Ayako Ijuin"2,3, Kazuma Ogiso², Yoshihiko Nishio3, \\ Michihisa Jougasaki ${ }^{*}$ \\ ${ }^{1}$ Institute for Clinical Research, National Hospital Organization Kagoshima Medical Center, Kagoshima, Japan \\ ${ }^{2}$ Department of Diabetes and Endocrine Medicine, National Hospital Organization Kagoshima Medical Center, \\ Kagoshima, Japan \\ ${ }^{3}$ Department of Diabetes and Endocrine Medicine, Graduate School of Medicine, Kagoshima University, \\ Kagoshima, Japan \\ Email: ${ }^{*}$ jougasaki@kagomc2.hosp.go.jp
}

Received 2 September 2015; accepted 27 October 2015; published 30 October 2015

Copyright (C) 2015 by authors and Scientific Research Publishing Inc.

This work is licensed under the Creative Commons Attribution International License (CC BY). http://creativecommons.org/licenses/by/4.0/

\section{Open Access}

\begin{abstract}
Background: There are few clinical trials addressing the difference in pleiotropic effects among dipeptidyl peptidase (DPP)-4 inhibitors. We aimed to identify difference in effects on biochemical markers of inflammation, oxidative stress, and atherosclerosis between two DPP-4 inhibitors in patients with type 2 diabetes. Methods: We prospectively observed twenty subjects with type 2 diabetes before and after a practical medication change from a treatment with pioglitazone and sitagliptin $50 \mathrm{mg}$ to a combination tablet containing the same dose of pioglitazone and alogliptin $25 \mathrm{mg}$, which was actually identical to switching from sitagliptin to alogliptin. After 3 months, changes from baseline in clinical data and various biochemical markers were evaluated. In particular, body mass index (BMI) and hemoglobin A1c (HbA1c) were additionally followed after 12 months for evaluation of chronic outcomes. Results: Among markers, serum levels of high molecular weight (HMW) adiponectin significantly increased from $6.9 \pm 3.6 \mu \mathrm{g} / \mathrm{ml}$ to $8.2 \pm 4.0 \mu \mathrm{g} / \mathrm{ml}(P$ $=0.0045$ ). Although no clinical data changed after 3 months, significant improvements in HbA1c and BMI were observed after 12 months. Their rates of changes tended to inversely correlate with the increased percentages of serum HMW adiponectin levels during initial 3 months, but they did
\end{abstract}

\footnotetext{
"Corresponding author.
}

How to cite this paper: Tokito, A., Koriyama, N., ljuin, A., Ogiso, K., Nishio, Y. and Jougasaki, M. (2015) Switching from Sitagliptin to Alogliptin under Treatment with Pioglitazone Increases High Molecular Weight Adiponectin in Type 2 Diabetes: A Prospective Observational Study. Journal of Diabetes Mellitus, 5, 258-266. http://dx.doi.org/10.4236/jdm.2015.54032 
not reach statistical significance. Conclusions: In spite of pretreatment with pioglitazone, additional increase in serum HMW adiponectin levels was demonstrated after switching from sitagliptin to alogliptin. Given multiple favorable roles of adiponectin in metabolic and cardiovascular states, alogliptin, at least when combined with pioglitazone, would be beneficial in treatment of type 2 diabetes.

\section{Keywords}

\section{Alogliptin, Dipeptidyl Peptidase-4 Inhibitor, High Molecular Weight Adiponectin, Type 2 Diabetes}

\section{Introduction}

Metabolic syndrome, which is characterized by visceral adiposity, is widely recognized as a risk factor for diverse metabolic disorders including type 2 diabetes, hypertension, and dyslipidemia, and finally causes atherosclerotic diseases. Although the molecular mechanisms underlying development or progression of these obesity-related comorbidities are not completely understood, growing evidence suggests that they are closely associated with states of inflammation and oxidative stress [1]-[3], both of which lead to adipose tissue dysfunction as well as vascular dysfunction. Therefore, using medicines with beneficial pleiotropic effects on adipose tissue or vasculature, such as suppressing inflammation or decreasing oxidative stress, would be more essential for the treatment of each metabolic disorder and protection of atherosclerosis.

Adipose tissue is an endocrine organ which secretes a number of so-called adipokines such as leptin, monocyte chemoattractant protein (MCP)-1, and adiponectin, and dysregulation of their productions seen in obese adipose tissue with chronic inflammation is involved in a variety of pathologic processes [4]. Among them, adiponectin is downregulated in various metabolic disorders [5] [6], and currently expected to be a promising pharmacological target for diabetes and metabolic syndrome because of its insulin sensitizing [7], anti-atherogenic [8], and anti-inflammatory actions [9]. There are three isoforms of adiponectin characterized as low, medium, and high molecular weight (HMW) adiponectin, and HMW adiponectin has been shown to be the most abundant and biologically active form of the hormone [10] [11]. Thus, upregulation of the HMW adiponectin levels might be a key to useful treatment of metabolic syndrome.

Dipeptidyl peptidase (DPP)-4 inhibitors are a recently emerged class of hypoglycemic agents that lower blood glucose concentrations in patients with type 2 diabetes by increasing active forms of incretin hormones such as glucagon-like peptide (GLP)-1 followed by amplification of glucose-dependent insulin secretion [12]. In addition to its insulinotropic action and well-known effect of pancreatic $\beta$ cell protection, GLP-1 has many biological properties including immune regulation, anti-inflammation, and cardiovascular protection [13]. On the other hand, DPP-4 is a serine protease widely expressed throughout the body and cleaves diverse range of bioactive proteins besides GLP-1, such as GLP-2, stromal cell-derived factor (SDF)-1, neuropeptide Y, and chemokines. Therefore, inhibition of its enzymatic actions by DPP-4 inhibitors has potential to exert various pleiotropic effects [14].

Although a variety of DPP-4 inhibitors with different chemical structures have ever been marketed, there is no clear evidence for difference in the pleiotropic effects among DPP-4 inhibitors. In the current study, herein, we prospectively observed patients with type 2 diabetes before and after switching DPP-4 inhibitor from sitagliptin to alogliptin under treatment with pioglitazone, and examined whether there were differences in various biochemical markers of inflammation, oxidative stress, and atherosclerosis as well as clinical statuses. Moreover, the correlations between the changes of these parameters and clinical data were analyzed.

\section{Methods}

\subsection{Study Participants}

Study subjects consecutively enrolled were outpatients with type 2 diabetes at the National Hospital Organization Kagoshima Medical Center in Japan. They had been treated with oral hypoglycemic drugs including both pioglitazone $15 \mathrm{mg}$ or $30 \mathrm{mg}$ and sitagliptin $50 \mathrm{mg}$ for more than 2 months at baseline. Inclusion criteria were age $\geq 20$ years, and hemoglobin $\mathrm{A} 1 \mathrm{c}(\mathrm{HbAlc}) \geq 6.0 \%$ despite lifestyle modifications and pharmacological in- 
terventions. Exclusion criteria were type 1 diabetes, parallel usage of insulin, and serum creatinine level $\geq 1.3$ $\mathrm{mg} / \mathrm{dl}$. Also excluded were those with current history of stroke, acute coronary syndrome, and any cardiovascular diseases requiring new treatment within 6 months or who had any malignancies or chronic inflammatory diseases.

\subsection{Study Design}

This study was an open-label, prospective, and non-comparative observational trial. The study protocol is summarized in Figure 1. The subjects were offered and approved a practical medication change from a treatment with pioglitazone and sitagliptin $50 \mathrm{mg}$ to a combination tablet containing the same dose of pioglitazone and alogliptin $25 \mathrm{mg}$ for reasons of economy and/or expediency, which is actually equal to switching from sitagliptin to alogliptin under treatment with pioglitazone. The doses of these two DPP-4 inhibitors are both conventional ones in Japan. Throughout the 12-month observation period, the patients were instructed to maintain the diet and exercise therapy. The pre-existing drugs such as hypoglycemic agents, anti-hypertensive drugs, and statins were unchanged, and none of new medications were added during the study. Clinical assessments and biochemical measurements were performed in all subjects before and after 3 months of the DPP-4 inhibitor switching. In particular, body mass index (BMI) and HbAlc were additionally followed at 12 months after switching for the evaluation of clinical outcomes in the chronic phase.

This observational study was performed between October 2011 and January 2013. The study protocol (Figure 1) was approved by the Institutional Review Board at the National Hospital Organization Kagoshima MedicalCenter and conducted in accordance with the Declaration of Helsinki and its amendments. After full explanation of this study, all subjects provided written informed consents.

\subsection{Outcome Measurements}

The clinical data included BMI, systolic and diastolic blood pressures, HbA1c, low density lipoprotein (LDL) cholesterol, high density lipoprotein (HDL) cholesterol, and triglyceride. In addition, we assessed various biochemical markers; HMW adiponectin, MCP-1, and highly sensitive C-reactive protein (Hs-CRP) as the inflammatory markers, total reactive oxygen species (ROS) and oxidized-LDL cholesterol as the oxidative stress markers, and asymmetric dimethylarginine (ADMA) and E-selectin as the atherosclerotic markers. All of them except for MCP-1 and E-selectin were measured at an independent central laboratory (SRL Hachioji Laboratory, Tokyo, Japan). Venous blood samples were taken in the morning after overnight fasting from each patient under sterile conditions. Serum and plasma samples were obtained by centrifugation and stored at $-80^{\circ} \mathrm{C}$ until analyses. Serum HMW adiponectin level was determined using a chemiluminescent enzyme immunoassay (Fuji Rebio, Tokyo, Japan). Plasma levels of MCP-1 and E-selectin were determined using each enzyme-linked immunosorbent assay (ELISA) (R\&D systems, Inc., Minneapolis, USA). Serum Hs-CRP level was measured using the latex agglutination nephelometry method (Siemens Healthcare Diagnostics, Inc., Marburg, Germany). Total ROS level in serum was measured using N, N-diethylparaphenylendiamine reaction method (SRL Hachioji Laboratory, Tokyo, Japan). Serum oxidized -LDL cholesterol level was measured using ELISA (SEKISUI MEDICAL Co., Tokyo, Japan). Serum ADMA level was determined using high performance liquid chromatography (SRL Hachioji Laboratory, Tokyo, Japan).

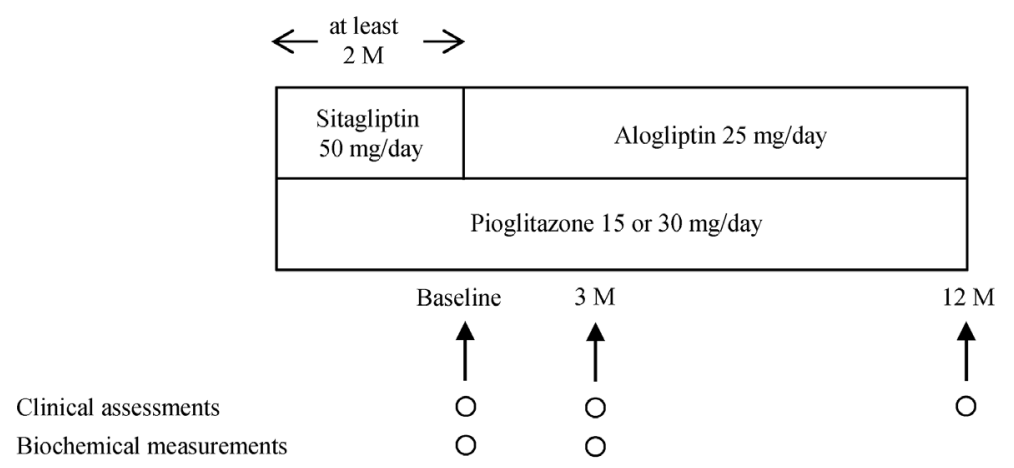

Figure 1. Study protocol. M, months. 


\subsection{Statistical Analysis}

Statistical analyses were performed using the StatView program for Macintosh (version 4.5-J; SAS Institute, Cary, NC). We used the paired $t$-test or nonparametric Wilcoxon signed-rank test for comparisons between baseline and 3- or 12-month follow-up data. Correlation analyses were performed using Spearman correlation coefficient. All data are presented as mean \pm standard deviation, and $P<0.05$ was considered statistically significant.

\section{Results}

\subsection{Baseline Characteristics of the Study Subjects}

A total of 20 subjects were enrolled in the current study, and none of them withdrew over the 12-month observation period, indicating that there were no adverse events caused by just switching of DPP-4 inhibitor from one to another. Baseline characteristics of the subjects and their pretreatment of hypoglycemic drugs at entering this study are shown in Table 1.

\subsection{Differences in Clinical Data and Various Markers between Baseline and 3 Months after Switching from Sitagliptin to Alogliptin}

At 3 months after switching, no clinical data including BMI and HbAlc changed from baseline (Table 2). Among various biochemical markers, significant increase from baseline value was observed only in serum HMW adiponectin levels $(6.9 \pm 3.6 \mu \mathrm{g} / \mathrm{ml}$ to $8.2 \pm 4.0 \mu \mathrm{g} / \mathrm{ml} ; P=0.0045)$ (Figure 2). None of the other biochemical markers including MCP-1, Hs-CRP, total ROS, oxidized-LDL cholesterol, ADMA, or E-selectin changed (Table 2). These findings indicate that serum HMW adiponectin levels might increase through the mechanisms independent of inflammation, oxidative stress, atherosclerotic risks, and clinical statuses of the study subjects.

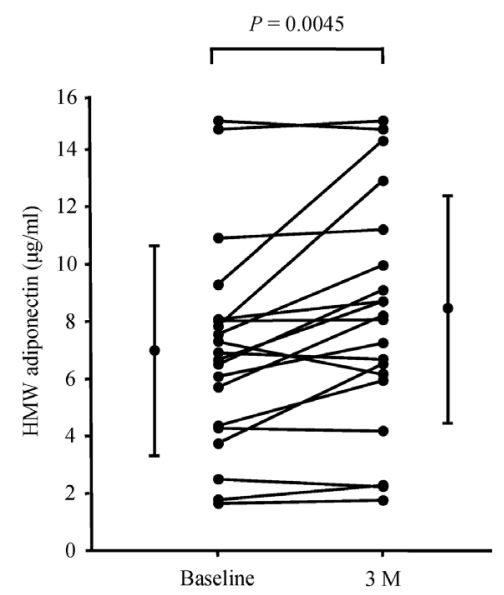

Figure 2. Individual changes in HMW adiponectin levels from baseline to 3 months after DPP-4inhibitorswitching.Bars indicate means \pm standard deviation. Wilcoxon signed-rank test $(n=20)$. HMW, high molecular weight; $M$, months.

Table 1. Baseline characteristics of the study subjects $(n=20)$.

\begin{tabular}{cc}
\hline Characteristics & Values \\
\hline Age (years) & $61.0 \pm 8.8$ \\
Sex (male $/$ female) & $17 / 3$ \\
BMI $\left(\mathrm{kg} / \mathrm{m}^{2}\right)$ & $26.7 \pm 3.0$ \\
Diabetes duration (years) & $8 \pm 5$ \\
HbAlc $(\%)$ & $7.1 \pm 0.6$ \\
Anti-hypertensive drugs & 16 \\
Statins & 11 \\
Pioglitazone $(15 / 30 \mathrm{mg})$ & $16 / 4$ \\
Other hypoglycemic drugs (SU/Met $/ \alpha \mathrm{GI})$ & $5 / 10 / 3$ \\
\hline
\end{tabular}

Values are the means \pm standard deviation or numbers. $\alpha \mathrm{GI}$, alpha glucosidase inhibitor; BMI, body mass index; HbA1c, hemoglobin A1c; Met, metformin; SU, sulfonylurea. 
Table 2. Clinical data and various biochemical markers at baseline and 3 months after DPP-4 inhibitor switching.

\begin{tabular}{|c|c|c|c|}
\hline Variables & Baseline & After $3 \mathrm{M}$ & $P$ \\
\hline \multicolumn{4}{|l|}{ Clinical data } \\
\hline $\mathrm{BMI}\left(\mathrm{kg} / \mathrm{m}^{2}\right)$ & $26.7 \pm 3.0$ & $26.8 \pm 2.9$ & NS \\
\hline $\mathrm{sBP}(\mathrm{mmHg})$ & $130 \pm 14$ & $126 \pm 16$ & NS \\
\hline $\mathrm{dBP}(\mathrm{mmHg})$ & $73 \pm 8$ & $74 \pm 10$ & NS \\
\hline $\operatorname{HbA} 1 \mathrm{c}(\%)$ & $7.1 \pm 0.6$ & $7.2 \pm 0.7$ & NS \\
\hline LDL cholesterol (mg/dl) & $104 \pm 20$ & $108 \pm 21$ & NS \\
\hline HDL cholesterol (mg/dl) & $55 \pm 13$ & $58 \pm 14$ & NS \\
\hline Triglyceride (mg/dl) & $119 \pm 39$ & $133 \pm 78$ & NS \\
\hline \multicolumn{4}{|l|}{ Markers } \\
\hline $\mathrm{MCP}-1$ (pg/mL) & $188 \pm 111$ & $188 \pm 98$ & NS \\
\hline Hs-CRP (ng/mL) & $644 \pm 634$ & $722 \pm 877$ & NS \\
\hline Total ROS (Unit) & $188 \pm 50$ & $181 \pm 30$ & NS \\
\hline Oxidized-LDL cholesterol (U/L) & $125 \pm 30$ & $134 \pm 27$ & NS \\
\hline ADMA (nmol/mL) & $0.42 \pm 0.05$ & $0.41 \pm 0.06$ & NS \\
\hline E-selectin (ng/mL) & $50.6 \pm 23.5$ & $51.0 \pm 19.8$ & NS \\
\hline
\end{tabular}

Values are the means \pm standard deviation. ADMA, asymmetric dimethylarginine; BMI, body mass index; dBP, diastolic blood pressure; HbA1c, hemoglobin Alc; HDL, high density lipoprotein; Hs-CRP, highly sensitive C-reactive protein; LDL, low density lipoprotein; M, months; MCP-1, monocyte chemoattractant protein-1; NS, not significant; ROS, reactive oxygen species; sBP, systolic blood pressure.

\subsection{Differences in HbA1c and BMI between Baseline and 12 Months after Switching from Sitagliptin to Alogliptin}

To assess the chronic effects of switching from sitagliptin to alogliptin under treatment with pioglitazone, we evaluated the changes of $\mathrm{HbA} 1 \mathrm{c}$ and BMI from baseline to 12 months after switching. Of note, both $\mathrm{HbA} 1 \mathrm{c}$ and BMI after 12 months slightly but significantly improved $(7.1 \% \pm 0.6 \%$ to $6.8 \% \pm 0.6 \%, P=0.013$, and $26.7 \pm$ $3.0 \mathrm{~kg} / \mathrm{m}^{2}$ to $26.2 \pm 3.3 \mathrm{~kg} / \mathrm{m}^{2}, P=0.047$; respectively) (Figure 3(a) and Figure 3(b)). Therefore, we further examined correlations between the increased percentages of plasma HMW adiponectin levels during initial 3 months $(\triangle \mathrm{HMW}$ adiponectin) and changes in $\mathrm{HbAlc}(\triangle \mathrm{HbAlc})$ or $\mathrm{BMI}(\triangle \mathrm{BMI})$ from baseline during 12 months after switching from sitagliptin to alogliptin $(n=15)$. As shown in Figure 3(c) and Figure 3(d), there tended to be modest inverse correlations both between $\triangle \mathrm{HMW}$ adiponectin and $\triangle \mathrm{HbAl \textrm {c }}$ and between $\triangle \mathrm{HMW}$ adiponectin and $\Delta \mathrm{BMI}(r=-0.339$ and -0.320 , respectively $)$, but neither of them reached statistical significance.

\section{Discussion}

In the current prospective study, the switching of DPP-4 inhibitor from sitagliptin to alogliptin under treatment with pioglitazone had a beneficial effect on adipocytes, in particular increasing the serum levels of HMW adiponectin in patients with type 2 diabetes. This pleiotropic effect was seen after 3 months of the drug switching without alterations in other biochemical markers of inflammation, oxidative stress, and atherosclerosis as well as body weight and blood glucose controls. At the later 12-month follow-up point, both HbAlc and BMI significantly decreased from baseline, and their rates of changes tended to inversely correlate with the increased percentages of serum HMW adiponectin levels during initial 3 months of this study. Until now, there are just a few clinical studies addressing the difference in pleiotropic effects among DPP-4 inhibitors [15]-[17]. Notably, a most recent study strongly supports our observation that switching from sitagliptin to vildagliptin, another DPP-4 inhibitor, significantly increased serum adiponectin levels after 3 months [17].

Pioglitazone, a thiazolidinedione class of hypoglycemic drugs, has been reported to increase serum levels of both total and HMW adiponectin by approximately 3 -fold in patients with type 2 diabetes [18]-[20]. As expected, at baseline in the present study when the subjects had already received pioglitazone-based medications at least for 2 months, serum HMW adiponectin levels were comparably increased [20]. It is worthy of note that just switching of DPP-4 inhibitor from sitagliptin to alogliptin for 3 months led to further elevation of serum levels 


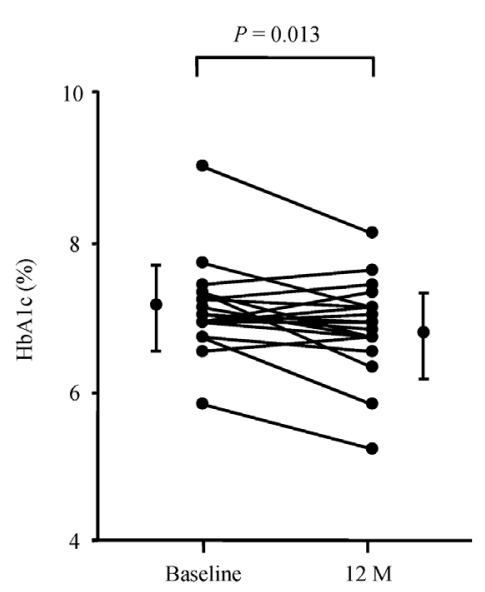

(a)

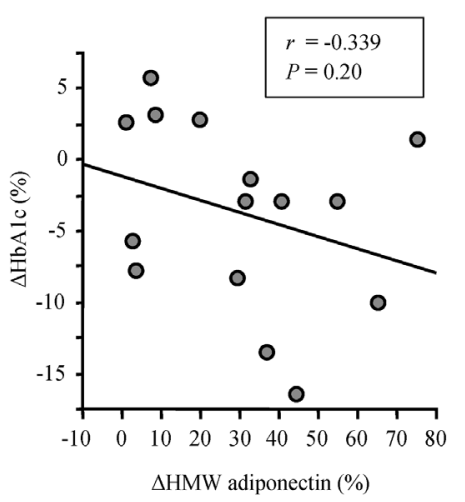

(c)

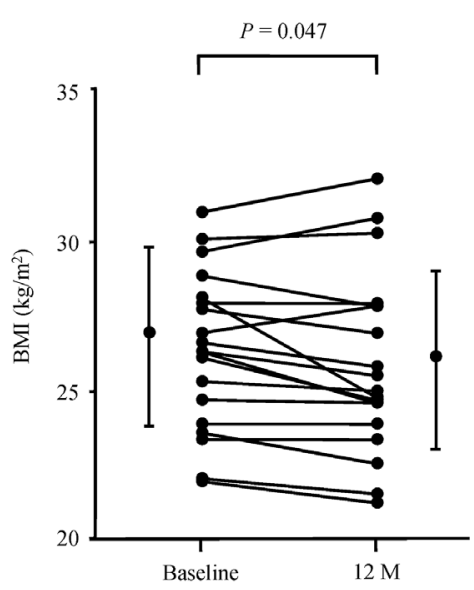

(b)

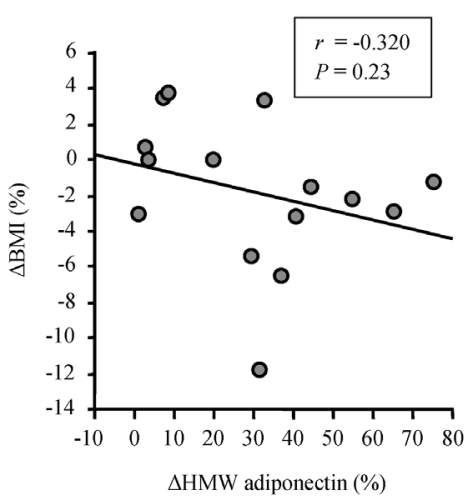

(d)

Figure 3. Individual changes of (a) HbAlc and (b) BMI from baseline to 12 months after switching from sitagliptin to alogliptin. Bars indicate means \pm standard deviation. Paired $t$-test $(n=20)$. Correlations of the change rates of $(c) H b A 1 c$ $(\triangle \mathrm{HbA} 1 \mathrm{c})$ and $(\mathrm{d}) \mathrm{BMI}(\triangle \mathrm{BMI})$ during 12 months with the increased percentages of HMW adiponectin during initial 3 months ( $\triangle$ HMW adiponectin) after switching from sitagliptin to alogliptin. Spearman correlation coefficient $(n=15)$. BMI, body mass index; HbA1c, hemoglobin A1c; HMW, high molecular weight; M, months.

of HMW adiponectin beyond baseline. To our knowledge, no previous report has demonstrated such an additional increase in serum levels of HMW adiponectin in patients already taking pioglitazone by using other hypoglycemic agents. As a large body of evidence indicates that adiponectin has systemic pleiotropic effects that are beneficial for insulin sensitivity and inflammation [7] [9], the present study suggests that the combination therapy with pioglitazone and alogliptin might be more protective against organ disorders including atherosclerosis than the monotherapy with pioglitazone.

The mechanism responsible for the additional increase in the serum HMW adiponectin levels by switching from sitagliptin to alogliptin remains unclear. After 3 months of the dug switching, no significant differences in clinical data or various biochemical markers except for the serum HMW adiponectin levels were observed (Table 2), indicating that the serum HMW adiponectin levels increased through mechanisms independent of inflammation, oxidative stress, and metabolic statuses. One possibility is that differences in selectivity and inhibitory activity for DPP-4 between alogliptin and sitagliptin might contribute to the additional increase in serum HMW adiponectin concentrations. Actually, alogliptin has been reported to have higher selectivity and stronger inhibitory activity for DPP-4 as compared with sitagliptin [21]. The powerful DPP-4 inhibition by alogliptin is expected to provide greater elevations of substrates for DPP-4 such as GLP-1 and SDF-1, some of which reportedly have various effects beyond glycemic control [13] [15]. Notably, a GLP-1 receptor agonist, exendin-4, has shown to directly induce the adiponectin expression in 3T3-L1 adipocyte through protein kinase A pathway [22]. 
In addition, benefit from the combination with pioglitazone and alogliptin should be considered as a potential contributor to the upregulation of HMW adiponectin. Of interest, a recent study showed a synergistic effect of co-treatment with pioglitazone and alogliptin on thermogenesis in beige/brown fat cells resulting in significant reduction of fat mass in mice, whereas each drug alone was ineffective [23]. As there is an inverse correlation between fat mass and HMW adiponectin [24], such synergistic effect of the combination therapy with pioglitazone and alogliptin might be a reason for the increased levels of HMW adiponectin observed here. To clarify whether pioglitazone synergistically contributed to additional increase of HMW adiponectin levels after switching, further full study comparing the effect of each DPP-4 inhibitor with and without pioglitazone is necessary.

Unfortunately, in the present study, we could not statistically certify clinical implications of the increase in serum HMW adiponectin level by switching from sitagliptin to alogliptin. However, given the modest trends of inverse relationships between $\triangle \mathrm{HMW}$ adiponectin and $\triangle \mathrm{HbA} 1 \mathrm{c}$ and between $\triangle \mathrm{HMW}$ adiponectin and $\Delta \mathrm{BMI}$, it is an attractive speculation that the improvements in $\mathrm{HbA} 1 \mathrm{c}$ and $\mathrm{BMI}$ at the end of this study might be derived from the prior upregulation of serum HMW adiponectin levels. In many previous reports, administration of recombinant adiponectin has shown beneficial effects on body weight and hyperglycemia [25]. Yamamoto et al. reported a longitudinal study showing that if baseline adiponectin level is high, insulin sensitivity improves in subsequent 2 years [26]. If plasma insulin levels decrease as a result of the improvement of systemic insulin sensitivity by the upregulation of HMW adiponectin, BMI might subsequently decrease. Further examination by a longer-term study including evaluation of the insulin sensitivity such as the homeostasis model assessment of insulin resistance is required to clarify this attractive speculation.

There are several limitations in this study. First, the current study was a non-comparative observational trial. Comparison with placebo-control group or cross-over design is needed in the future investigation to confirm our results. Second, we did not consider drug adherence and life style of the study subjects during the observation. Changes in these factors can affect body weight or blood glucose level. But in the current study, upregulation of HMW adiponectin levels was not along with the improvements in BMI and HbAlc at 3 months after the drug switching, indicating that the HMW adiponectin upregulation may have less to do with these potential confounding factors. Finally, as mentioned above, we could not statistically certify the clinical implications of the increase in serum HMW adiponectin level by switching from sitagliptin to alogliptin.

\section{Conclusion}

In summary, in spite of pretreatment with pioglitazone, additional increase in serum levels of HMW adiponectin was demonstrated a few months after switching from sitagliptin to alogliptin. Although the large-scale, longterm, and placebo-controlled study must be conducted to identify the underlying mechanisms and the clinical implications of our results, we speculated that this study might provide a new insight into the difference in pleiotropic effects among DPP-4 inhibitors, and that alogliptin, at least when combined with pioglitazone, would be beneficial in the treatment of type 2 diabetes.

\section{Acknowledgements}

The present work was supported by a grant from the National Hospital Organization Collaborative Clinical Research. We thank Reiko Saino for secretarial work and Yoko Takenoshita for technical assistance.

\section{References}

[1] Hotamisligil, G.S. (2006) Inflammation and Metabolic Disorders. Nature, 444, 860-867. http://dx.doi.org/10.1038/nature05485

[2] Palmieri, V.O., Grattagliano, I., Portincasa, P. and Palasciano, G. (2006) Systemic Oxidative Alterations Are Associated with Visceral Adiposity and Liver Steatosis in Patients with Metabolic Syndrome. The Journal of Nutrition, 136, 3022-3026.

[3] Rocha, V.Z. and Libby, P. (2009) Obesity, Inflammation, and Atherosclerosis. Nature Reviews Cardiology, 6, 399-409. http://dx.doi.org/10.1038/nrcardio.2009.55

[4] Matsuzawa, Y., Funahashi, T. and Nakamura, T. (1999) Molecular Mechanism of Metabolic Syndrome X: Contribution of Adipocytokines Adipocyte-Derived Bioactive Substances. Annals of the New York Academy of Sciences, 892, 146-154. http://dx.doi.org/10.1111/j.1749-6632.1999.tb07793.x

[5] Arita, Y., Kihara, S., Ouchi, N., Takahashi, M., Maeda, K., Miyagawa, J., et al. (1999) Paradoxical Decrease of an 
Adipose-Specific Protein, Adiponectin, in Obesity. Biochemical and Biophysical Research Communications, 257, 7983. http://dx.doi.org/10.1006/bbrc.1999.0255

[6] Hotta, K., Funahashi, T., Arita, Y., Takahashi, M., Matsuda, M., Okamoto, Y., et al. (2000) Plasma Concentrations of a Novel, Adipose-Specific Protein, Adiponectin, in Type 2 Diabetic Patients. Arteriosclerosis, Thrombosis, and Vascular Biology, 20, 1595-1599. http://dx.doi.org/10.1161/01.ATV.20.6.1595

[7] Yamauchi, T., Kamon, J., Waki, H., Terauchi, Y., Kubota, N., Hara, K., et al. (2001) The Fat-Derived Hormone Adiponectin Reverses Insulin Resistance Associated with Both Lipoatrophy and Obesity. Nature Medicine, 7, 941-946. http://dx.doi.org/10.1038/90984

[8] Okamoto, Y., Arita, Y., Nishida, M., Muraguchi, M., Ouchi, N., Takahashi, M., et al. (2000) An Adipocyte-Derived Plasma Protein, Adiponectin, Adheres to Injured Vascular Walls. Hormone and Metabolic Research = Hormon- und Stoffwechselforschung= Hormones et metabolisme, 32, 47-50. http://dx.doi.org/10.1055/s-2007-978586

[9] Ouchi, N., Kihara, S., Arita, Y., Okamoto, Y., Maeda, K., Kuriyama, H., et al. (2000) Adiponectin, an Adipocyte-Derived Plasma Protein, Inhibits Endothelial Nf-Kappab Signaling through a Camp-Dependent Pathway. Circulation, 102, 1296-1301. http://dx.doi.org/10.1161/01.CIR.102.11.1296

[10] Hara, K., Horikoshi, M., Yamauchi, T., Yago, H., Miyazaki, O., Ebinuma, H., et al. (2006) Measurement of the High-Molecular Weight Form of Adiponectin in Plasma Is Useful for the Prediction of Insulin Resistance and Metabolic Syndrome. Diabetes Care, 29, 1357-1362. http://dx.doi.org/10.2337/dc05-1801

[11] Lara-Castro, C., Luo, N., Wallace, P., Klein, R.L. and Garvey, W.T. (2006) Adiponectin Multimeric Complexes and the Metabolic Syndrome Trait Cluster. Diabetes, 55, 249-259. http://dx.doi.org/10.2337/diabetes.55.01.06.db05-1105

[12] Drucker, D.J. and Nauck, M.A. (2006) The Incretin System: Glucagon-Like Peptide-1 Receptor Agonists and Dipeptidyl Peptidase-4 Inhibitors in Type 2 Diabetes. The Lancet, 368, 1696-1705. http://dx.doi.org/10.1016/S0140-6736(06)69705-5

[13] Campbell, J.E. and Drucker, D.J. (2013) Pharmacology, Physiology, and Mechanisms of Incretin Hormone Action. Cell Metabolism, 17, 819-837. http://dx.doi.org/10.1016/j.cmet.2013.04.008

[14] Zhao, Y., Yang, L. and Zhou, Z. (2014) Dipeptidyl Peptidase-4 Inhibitors: Multitarget Drugs, Not Only Antidiabetes Drugs. Journal of Diabetes, 6, 21-29. http://dx.doi.org/10.1111/1753-0407.12063

[15] Fujita, H., Taniai, H., Murayama, H., Ohshiro, H., Hayashi, H., Sato, S., et al. (2014) Dpp-4 Inhibition with Alogliptin on Top of Angiotensin Ii Type 1 Receptor Blockade Ameliorates Albuminuria via Up-Regulation of Sdf- $1 \alpha$ in Type 2 Diabetic Patients with Incipient Nephropathy. Endocrine Journal, 61, 159-166. http://dx.doi.org/10.1507/endocrj.EJ13-0305

[16] Rizzo, M.R., Barbieri, M., Marfella, R. and Paolisso, G. (2012) Reduction of Oxidative Stress and Inflammation by Blunting Daily Acute Glucose Fluctuations in Patients with Type 2 Diabetes: Role of Dipeptidyl Peptidase-IV Inhibition. Diabetes Care, 35, 2076-2082. http://dx.doi.org/10.2337/dc12-0199

[17] Takeshita, Y., Takamura, T., Kita, Y., Otoda, T., Kato, K., Wakakuri, H., et al. (2015) Vildagliptin vs. Liraglutide as a Second-Line Therapy Switched from Sitagliptin-Based Regimens in Patients with Type 2 Diabetes: A Randomized, Parallel-Group Study. Journal of Diabetes Investigation, 6, 192-200. http://dx.doi.org/10.1111/jdi.12269

[18] Hirose, H., Kawai, T., Yamamoto, Y., Taniyama, M., Tomita, M., Matsubara, K., et al. (2002) Effects of Pioglitazone on Metabolic Parameters, Body Fat Distribution, and Serum Adiponectin Levels in Japanese Male Patients with Type 2 Diabetes. Metabolism: Clinical and Experimental, 51, 314-317. http://dx.doi.org/10.1053/meta.2002.30506

[19] Tonelli, J., Li, W., Kishore, P., Pajvani, U.B., Kwon, E., Weaver, C., et al. (2004) Mechanisms of Early Insulin-Sensitizing Effects of Thiazolidinediones in Type 2 Diabetes. Diabetes, 53, 1621-1629. http://dx.doi.org/10.2337/diabetes.53.6.1621

[20] Aso, Y., Yamamoto, R., Suetsugu, M., Matsumoto, S., Wakabayashi, S., Matsutomo, R., et al. (2007) Comparison of the Effects of Pioglitazone and Voglibose on Circulating Total and High-Molecular-Weight Adiponectin, and on Two Fibrinolysis Inhibitors, in Patients with Type 2 Diabetes. Diabetic Medicine: A Journal of the British Diabetic Association, 24, 962-968. http://dx.doi.org/10.1111/j.1464-5491.2007.02204.X

[21] Nabeno, M., Akahoshi, F., Kishida, H., Miyaguchi, I., Tanaka, Y., Ishii, S., et al. (2013) A Comparative Study of the Binding Modes of Recently Launched Dipeptidyl Peptidase IV Inhibitors in the Active Site. Biochemical and Biophysical Research Communications, 434, 191-196. http://dx.doi.org/10.1016/j.bbrc.2013.03.010

[22] Kim Chung le, T., Hosaka, T., Yoshida, M., Harada, N., Sakaue, H., Sakai, T., et al. (2009) Exendin-4, a Glp-1 Receptor Agonist, Directly Induces Adiponectin Expression through Protein Kinase a Pathway and Prevents Inflammatory Adipokine Expression. Biochemical and Biophysical Research Communications, 390, 613-618. http://dx.doi.org/10.1016/i.bbrc.2009.10.015

[23] Masuda, T., Fu, Y., Eguchi, A., Czogalla, J., Rose, M.A., Kuczkowski, A., et al. (2014) Dipeptidyl Peptidase IV Inhibitor Lowers Ppargamma Agonist-Induced Body Weight Gain by Affecting Food Intake, Fat Mass, and Beige/Brown 
Fat but Not Fluid Retention. American Journal of Physiology Endocrinology and Metabolism, 306, E388-E398. http://dx.doi.org/10.1152/ajpendo.00124.2013

[24] Indulekha, K., Surendar, J., Anjana, R.M., Gokulakrishnan, K., Balasubramanyam, M., Aravindhan, V., et al. (2012) Circulating Levels of High Molecular Weight (HMW) Adiponectin and Total Adiponectin in Relation to Fat Distribution, Oxidative Stress and Inflammation in Asian Indians. Disease Markers, 33, 185-192. http://dx.doi.org/10.1155/2012/672939

[25] Turer, A.T. and Scherer, P.E. (2012) Adiponectin: Mechanistic Insights and Clinical Implications. Diabetologia, 55, 2319-2326. http://dx.doi.org/10.1007/s00125-012-2598-x

[26] Yamamoto, Y., Hirose, H., Saito, I., Nishikai, K. and Saruta, T. (2004) Adiponectin, an Adipocyte-Derived Protein, Predicts Future Insulin Resistance: Two-Year Follow-Up Study in Japanese Population. The Journal of Clinical Endocrinology and Metabolism, 89, 87-90. http://dx.doi.org/10.1210/jc.2003-031163 